\title{
COMPLEXITY OF A DISJOINT MATCHING PROBLEM ON BIPARTITE GRAPHS
}

\author{
GREGORY J. PULEO
}

\begin{abstract}
We consider the following question: given an $(X, Y)$-bigraph $G$ and a set $S \subseteq X$, does $G$ contain two disjoint matchings $M_{1}$ and $M_{2}$ such that $M_{1}$ saturates $X$ and $M_{2}$ saturates $S$ ? When $|S| \geq|X|-1$, this question is solvable by finding an appropriate factor of the graph. In contrast, we show that when $S$ is allowed to be an arbitrary subset of $X$, the problem is NP-hard.
\end{abstract}

\section{INTRODUCTION}

A matching in a graph $G$ is a set of pairwise disjoint edges. A matching covers a vertex $v \in V(G)$ if $v$ lies in some edge of the matching, and a matching saturates a set $S \subseteq V(G)$ if it covers every vertex of $S$.

An $(X, Y)$-bigraph is a bipartite graph with partite sets $X$ and $Y$. The fundamental result of matching theory is Hall's Theorem [5], which states that an $(X, Y)$-bigraph contains a matching that saturates $X$ if and only if $|N(S)| \geq|S|$ for all $S \subseteq X$. While Hall's Theorem does not immediately suggest an efficient algorithm for finding a maximum matching, such algorithms have been discovered and are well-known [1, 6].

A natural way to extend Hall's Theorem is to ask for necessary and sufficient conditions under which multiple disjoint matchings can be found. This approach was taken by Lebensold, who obtained the following generalization of Hall's Theorem.

Theorem 1.1 (Lebensold 9]). An (X,Y)-bigraph has $k$ disjoint matchings, each saturating $X$, if and only if

$$
\sum_{y \in Y} \min \{k,|N(y) \cap S|\} \geq k|S|
$$

for all $S \subseteq X$.

When $k=1$, the left side of (1D) is just $|N(S)|$, so Theorem 1.1 contains Hall's Theorem as a special case. As observed by Brualdi, Theorem 1.1 is equivalent to a theorem of Fulkerson [3] about disjoint permutations of 0,1-matrices. Theorem 1.1 is also a special case of Lovasz's $(g, f)$-factor theorem [10. Like Hall's Theorem, Theorem 1.1 does not immediately suggest an efficient algorithm, but efficient algorithms exist for solving the $(g, f)$-factor problem [4, and these algorithms can be applied to find the desired $k$ disjoint matchings. We discuss the algorithmic aspects further in Section 4.

A different extension was considered by Frieze [2, who considered the following problem:

Disjoint Matchings (DM)

Input: Two $(X, Y)$-bigraphs $G_{1}, G_{2}$ on the same vertex set. 
Question: Are there matchings $M_{1} \subseteq G_{1}, M_{2} \subseteq G_{2}$ such that $M_{1} \cap M_{2}=\emptyset$ and each $M_{i}$ saturates $X$ ?

When $G_{1}=G_{2}$, this problem is just the $k=2$ case of the problem considered by Lebensold, and is therefore polynomially solvable. On the other hand, Frieze proved that the Disjoint Matchings problem is NP-hard in general.

In this paper, we consider the following disjoint-matching problem, which can be naturally viewed as a restricted case of the Disjoint Matchings problem:

\section{Single-Graph Disjoint Matchings (SDM)}

Input: An $(X, Y)$-bigraph $G$ and a vertex set $S \subseteq X$.

Question: Are there matchings $M_{1}, M_{2} \subseteq G$ such that $M_{1} \cap M_{2}=$

$\emptyset, M_{1}$ saturates $X$, and $M_{2}$ saturates $S$ ?

We call such a pair $\left(M_{1}, M_{2}\right)$ an $S$-pair. When $S=X$, this problem is also equivalent to the $k=2$ case of Lebensold's problem. The problem SDM is similar to a problem considered by Kamalian and Mkrtchyan [7, who proved that the following problem is NP-hard:

\section{Residual Matching}

Input: An $(X, Y)$-bigraph $G$ and a nonnegative integer $k$.

Question: Are there matchings $M_{1}, M_{2} \subseteq G$ such that $M_{1} \cap M_{2}=$

$\emptyset, M_{1}$ is a maximum matching, and $\left|M_{2}\right| \geq k$ ?

When $G$ has a perfect matching, we can think of the Residual Matching problem as asking whether there is some $S \subseteq X$ with $|S|=k$ such that $G$ has an $S$-pair. In contrast, the SDM problem asks whether some particular $S$ admits an $S$-pair. Since $k$ is part of the input to the Residual Matching problem, it is a priori possible that SDM could be polynomially solvable while the Residual Matching problem is NP-hard, since one might need to check exponentially many candidate sets $S$.

In Section 2 we give a quick reduction from SDM to DM, justifying the view of SDM as a special case of DM, and in Section 3 we show that SDM is NPhard, thereby strengthening Frieze's result. In Section 4 we show that SDM is polynomially solvable under the additional restriction $|S| \geq|X|-1$.

\section{REDUCing SDM to DM}

In this section, we show that any instance of SDM with $|S|<|X|-1$ reduces naturally to an instance of DM. Since SDM-instances with $|S| \geq|X|-1$ are polynomially solvable, as we show in Section 4 , this justifies the claim that SDM is a special case of DM.

Theorem 2.1. Let $G$ be an $(X, Y)$-bigraph and let $S \subseteq V(G)$ with $|S|<|X|-1$. Construct graphs $G_{1}, G_{2}$ as follows:

$$
\begin{aligned}
& V\left(G_{1}\right)=V\left(G_{2}\right)=V(G), \\
& E\left(G_{1}\right)=E(G), \\
& E\left(G_{2}\right)=E(G) \cup\{x y: x \in X-S, y \in Y\} .
\end{aligned}
$$

The graph $G$ has an $S$-pair if and only if there are disjoint matchings $M_{1}, M_{2}$ contained in $G_{1}, G_{2}$ respectively, each saturating $X$.

Proof. If $|Y|<|X|$, then it is clear that $G$ has no $S$-pair and that $G_{1}, G_{2}$ do not have perfect matchings, so assume that $|Y| \geq|X|$. 
First suppose that $M_{1}, M_{2}$ are disjoint matchings contained in $G_{1}, G_{2}$ respectively, each saturating $X$. Let $M_{1}^{\prime}=M_{1}$ and let $M_{2}^{\prime}=\left\{e \in M_{2}: e \cap X \subseteq S\right\}$. It is clear that $\left(M_{1}^{\prime}, M_{2}^{\prime}\right)$ is an $S$-pair.

Now suppose that we are given an $S$-pair $\left(M_{1}^{\prime}, M_{2}^{\prime}\right)$. In order to obtain the matchings $M_{1}, M_{2}$ in $G_{1}, G_{2}$ as needed, we need to enlarge $M_{2}^{\prime}$ so that it saturates all of $X$, rather than only saturating $S$. Let $Y^{\prime}=\left\{y \in Y: y \notin V\left(M_{2}^{\prime}\right)\right\}$, and let $H=G_{2}\left[(X-S) \cup Y^{\prime}\right]-M_{1}^{\prime}$.

We claim that $H$ has a matching that saturates $X-S$, and prove this by verifying Hall's Condition. Let any $X_{0} \subseteq X-S$ be given. If $\left|X_{0}\right|=1$, say $X_{0}=\left\{x_{0}\right\}$, then $N_{H}\left(X_{0}\right)$ contains all of $Y^{\prime}$ except possibly the mate of $x_{0}$ in $M_{1}$. Hence

$$
\left|N_{H}\left(X_{0}\right)\right| \geq\left|Y^{\prime}\right|-1=|Y|-|S|-1 \geq|X|-|S|-1 \geq 1=\left|X_{0}\right|,
$$

as desired. On the other hand, if $\left|X_{0}\right| \geq 2$, then $N_{H}\left(X_{0}\right)$ contains all of $Y^{\prime}$, so that

$$
\left|N_{H}\left(X_{0}\right)\right|=\left|Y^{\prime}\right|=|Y|-|S| \geq|X|-|S| \geq\left|X_{0}\right| \text {. }
$$

Hence Hall's Condition holds for $H$. Now let $M$ be a perfect matching in $H$, let $M_{1}=M_{1}^{\prime}$, and let $M_{2}=M_{2}^{\prime} \cup M$. By construction, $M_{2}$ is a matching in $G_{2}$ that saturates $X$. It is clear that $M_{1} \cap M_{2}=\emptyset$, since the edges in $M_{1}^{\prime}$ were omitted from $H$. Hence $M_{1}$ and $M_{2}$ are as desired.

\section{Finding Two Matchings is NP-Hard}

Given an instance $(G, S)$ of SDM, we call a pair of matchings $\left(M_{1}, M_{2}\right)$ satisfying the desired condition an $S$-pair. When $G^{\prime}$ is a subgraph of $G$ and $S^{\prime}=S \cap V\left(G^{\prime}\right)$, we say that an $S$-pair $\left(M_{1}, M_{2}\right)$ contains an $S^{\prime}$-pair $\left(M_{1}^{\prime}, M_{2}^{\prime}\right)$ if $M_{1}^{\prime} \subseteq M_{1}$ and $M_{2}^{\prime} \subseteq M_{2}$.

We prove that SDM is NP-hard via a reduction from 3SAT. Let $c_{1}, \ldots, c_{s}$ be the clauses and $\theta_{1}, \ldots, \theta_{t}$ be the variables of an arbitrary 3SAT instance. We define a graph $G$ as follows.

For each variable $\theta_{i}$, let $H_{i}$ be a copy of the cycle $C_{4 s}$, with vertices $v_{i, 1}, \ldots, v_{i, 4 s}$ written in order. Define

$$
\begin{aligned}
X_{i} & =\left\{v_{i, j}: j \text { is even }\right\}, \\
S_{i} & =\left\{v_{i, j}: j \equiv 2 \quad(\bmod 4)\right\} .
\end{aligned}
$$

Since $H_{i}$ is an even cycle, it has exactly two perfect matchings, one containing the edge $v_{i, 1} v_{i, 2}$ and the other containing the edge $v_{i, 2} v_{i, 3}$. In an $S_{i}$-pair $\left(M_{1}, M_{2}\right)$ for $H_{i}$, we have $v_{i, 1} v_{i, 2} \in M_{1}$ if and only if $v_{i, 2} v_{i, 3} \in M_{2}$, and the same argument holds for the other vertices of $S_{i}$. Thus, $H_{i}$ has only two possible $S_{i}$-pairs, illustrated in Figure 1. We call these pairs the true pair and false pair for $H_{i}$.

In the full graph $G$, we will not add any new edges incident to the vertices of $X_{i}$, so it will still be the case that any $S$-pair in the full graph induces either the true pair or the false pair in $H_{i}$. We use these pairs to encode the truth values of the corresponding 3SAT-variables.

For each clause $c_{k}$, let $L_{k}$ be a copy of $K_{2}$, with vertices $w_{k}, z_{k}$. Let $G=$ $\left(\bigcup_{j} H_{j}\right) \cup\left(\bigcup_{k} L_{k}\right)$. Add edges to $G$ as follows: if the variable $\theta_{i}$ appears positively in the clause $c_{k}$, add an edge from $w_{k}$ to $v_{i, 4 k-3}$, and if the variable $\theta_{i}$ appears negatively in the clause $c_{k}$, add an edge from $w_{k}$ to $v_{i, 4 k-1}$.

Let $X=\bigcup_{j}\left(X_{j} \cup\left\{w_{j}\right\}\right)$, and let $Y=V(G)-X$. Observe that $(X, Y)$ is a bipartition of $V(G)$. Let $S=\left(\bigcup S_{j}\right) \cup \bigcup\left\{w_{j}\right\}$. 


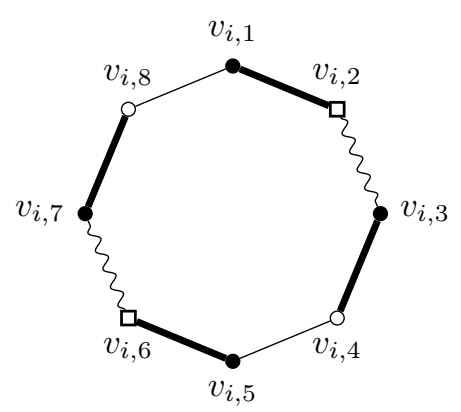

True pair.

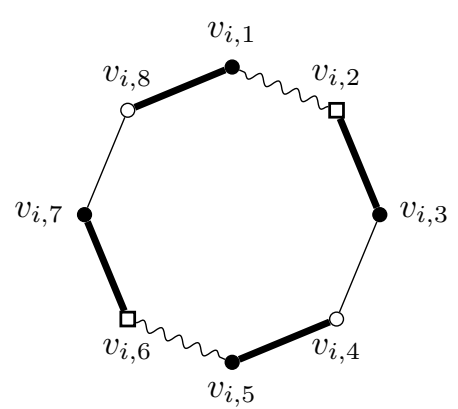

False pair.

Figure 1. True and false pairs for $H_{i}$ in the case $s=2$. White vertices lie in $X_{i}$; black vertices lie in $Y_{i}$; square vertices lie in $S_{i}$. Thick lines denote edges in $M_{1}$, wavy lines denote edges in $M_{2}$.

Lemma 3.1. G has an $S$-pair if and only if the given $3 S A T$ instance is satisfiable.

Proof. Let $\left(M_{1}, M_{2}\right)$ be an $S$-pair. We show that the 3SAT instance is satisfiable.

For any variable $\theta_{i}$, the vertices of $X \cap H_{i}$ have neighborhoods contained in $H_{i}$. Hence, $\left(M_{1}, M_{2}\right)$ contains an $S_{i}$-pair, and in particular contains either the true pair or the false pair for $H_{i}$. Construct an assignment by setting each variable $\theta_{i}$ to be true if $\left(M_{1}, M_{2}\right)$ contains the true pair for $H_{i}$ and false otherwise. We claim that this is a satisfying assignment.

Consider any clause $c_{k}$. Since $M_{1}$ is a perfect matching and $w_{k}$ is the only neighbor of $z_{k}$, we have $w_{k} z_{k} \in M_{1}$. Since $w_{k} \in S$, some edge $w_{k} v_{i, 4 k-3}$ or $w_{k} v_{i, 4 k-1}$ lies in $M_{2}$.

If $w_{k} v_{i, 4 k-1} \in M_{2}$, then $v_{i, 4 k-2} v_{i, 4 k-2} \notin M_{2}$, so the given $S$-pair contains the false pair for $H_{i}$. Since $w_{k} v_{i, 4 k-1} \in E(G)$, the clause $c_{k}$ contains a negative instance of $\theta_{i}$, so the constructed assignment satisfies the clause $c_{k}$. On the other hand, if $w_{k} v_{i, 4 k-3} \in M_{2}$, then the given $S$-pair contains the true pair for $H_{i}$ and $\theta_{i}$ appears postively in $w_{k}$, so we again see that $w_{k}$ is satisfied.

Conversely, suppose that the 3SAT problem has a satisfying assignment. Consider the pair of matchings $\left(M_{1}, M_{2}\right)$ in $G$ obtained as follows. For each variable $i$, add the true pair for each $H_{i}$ where $\theta_{i}$ is true and the false pair for each $H_{i}$ where $\theta_{i}$ is false. For each clause $c_{k}$, add the edge $w_{k} z_{k}$ to $M_{1}$. Choose some variable $\theta_{i}$ that satisfies the clause $c_{k}$. If $\theta_{i}$ is true, add the edge $w_{k} v_{i, 4 k-3}$ to $M_{2}$, and otherwise add $w_{k} v_{i, 4 k-1}$ to $M_{2}$. It is straightforward to check that this is an $S$-pair for $G$.

\section{Corollary 3.2. SDM is NP-hard.}

Viewing SDM as a special case of DM as in Section 2, we obtain the following NP-hardness result for DM.

Corollary 3.3. DM is NP-hard, even when restricted to instances for which $E\left(G_{1}\right) \subseteq$ $E\left(G_{2}\right)$.

\section{An Algorithm for the Case $|S| \geq|X|-1$}

In this section, we provide a polynomial-time algorithm for solving SDM in the special case $|S| \geq|X|-1$. Our algorithm requires the notion of a $(g, f)$-factor as well as the notion of edge coloring. 
Definition 4.1. If $G$ is a graph and $g$ and $f$ are functions from $V(G)$ into the nonnegative integers, a $(g, f)$-factor is a subgraph $H \subseteq G$ such that $g(v) \leq d_{H}(v) \leq$ $f(v)$ for all $v \in V(G)$.

Lovasz [10] gave a Hall-like condition for a graph to have a $g, f$-factor, and polynomial-time algorithms are known for determining whether such a factor exists (for example, [4). In the bipartite case we are considering here, the problem of determining whether such a factor exists can also be reduced to a feasible-flow problem.

Definition 4.2. For a nonnegative integer $k$, a $k$-edge coloring of a graph $G$ is a function $f: E(G) \rightarrow\{1, \ldots, k\}$ such that $f\left(e_{1}\right) \neq f\left(e_{2}\right)$ whenever $e_{1}, e_{2}$ are distinct edges sharing an endpoint. The edge-chromatic number of $G$, written $\chi^{\prime}(G)$, is the smallest integer $k$ such that $G$ has a $k$-edge-coloring.

Theorem 4.3 (König's line-coloring theorem [8]). If $G$ is a bipartite graph, then $\chi^{\prime}(G)=\Delta(G)$, where $\Delta(G)$ is the maximum degree of $G$.

Theorem 4.4. There is a polynomial-time algorithm to solve SDM restricted to instances for which $|S| \geq|X|-1$.

Proof. To avoid triviality, assume that $|X|>1$. Define functions $f$ and $g$ as follows.

$$
\begin{aligned}
& f(v)= \begin{cases}1, & \text { if } v \in X-S, \\
2, & \text { otherwise }\end{cases} \\
& g(v)= \begin{cases}f(v), & \text { if } v \in X, \\
0, & \text { otherwise }\end{cases}
\end{aligned}
$$

We can check in polynomial time whether $G$ has a $(g, f)$-factor. On the other hand, any $(g, f)$-factor $H$ has maximum degree 2 , and thus satisfies $\chi^{\prime}(H)=2$, by König's line-coloring theorem. Since $d_{H}(v)=2$ for all $v \in S$, any 2-edge-coloring of $H$ uses colors $\{1,2\}$ at each vertex of $S$. Furthermore, if $X-S \neq \emptyset$, then by switching colors if necessary, we can assume that the vertex in $X-S$ has only 1 as an incident color. Taking $M_{1}$ and $M_{2}$ to consist of the edges of color 1 and 2 respectively, we see that $\left(M_{1}, M_{2}\right)$ is an $S$-pair in $G$. Conversely, if $\left(M_{1}^{\prime}, M_{2}^{\prime}\right)$ is any $S$-pair in $G$, then $M_{1}^{\prime} \cup M_{2}^{\prime}$ is a $(g, f)$-factor.

Hence, $G$ has a $(g, f)$-factor if and only if $G$ has an $S$-pair, so checking for such a factor solves the problem in polynomial time.

For any fixed $k$, the problem SDM is polynomial-time solvable on instances with $|S| \leq k$ : we can iterate over the $O\left(|Y|^{k}\right)$ possible choices for $M_{2}$, and for each possible choice, check whether $G-M_{2}$ has a perfect matching $M_{1}$. Since the reduction in Section 3 produces SDM instances in which $|X-S|$ is arbitrarily large, Theorem 4.4 suggests that SDM might also be polynomially solvable when $|S|$ is bounded less strongly from below. However, the trick of using $(g, f)$-factors is no longer sufficient by itself to solve the problem when $k>1$.

Question 4.5. For fixed $k>1$, is there a polynomial-time algorithm to solve SDM on instances with $|S| \geq|X|-k$ ? 


\section{REFERENCES}

1. H. Alt, N. Blum, K. Mehlhorn, and M. Paul, Computing a maximum cardinality matching in a bipartite graph in time $O\left(n^{1.5} \sqrt{m / \log n}\right)$, Inform. Process. Lett. 37 (1991), no. 4, 237-240. MR 1095712 (91m:68141)

2. A. M. Frieze, Complexity of a 3-dimensional assignment problem, European J. Oper. Res. 13 (1983), no. 2, 161-164. MR 708379 (84i:68064)

3. D. R. Fulkerson, The maximum number of disjoint permutations contained in a matrix of zeros and ones, Canad. J. Math. 16 (1964), 729-735. MR 0168583 (29 \#5843)

4. Harold N. Gabow, An efficient reduction technique for degree-constrained subgraph and bidirected network flow problems, Proceedings of the Fifteenth Annual ACM Symposium on Theory of Computing (New York, NY, USA), STOC '83, ACM, 1983, pp. 448-456.

5. Philip Hall, On representatives of subsets, J. London Math. Soc 10 (1935), no. 1, 26-30.

6. John E. Hopcroft and Richard M. Karp, An $n^{5 / 2}$ algorithm for maximum matchings in bipartite graphs, SIAM J. Comput. 2 (1973), 225-231. MR 0337699 (49 \#2468)

7. R. R. Kamalian and V. V. Mkrtchyan, On complexity of special maximum matchings constructing, Discrete Math. 308 (2008), no. 10, 1792-1800. MR 2394447 (2009d:05197)

8. Dénes König, Graphok és alkalmazásuk a determinánsok és a halmazok elméletére, Mathematikai és Természettudományi Ertesito 34 (1916), 104-119.

9. Kenneth Lebensold, Disjoint matchings of graphs, J. Combinatorial Theory Ser. B 22 (1977), no. 3, 207-210. MR 0450138 (56 \#8435)

10. László Lovász, Subgraphs with prescribed valencies, J. Combinatorial Theory 8 (1970), 391416. MR 0265201 (42 \#113) 